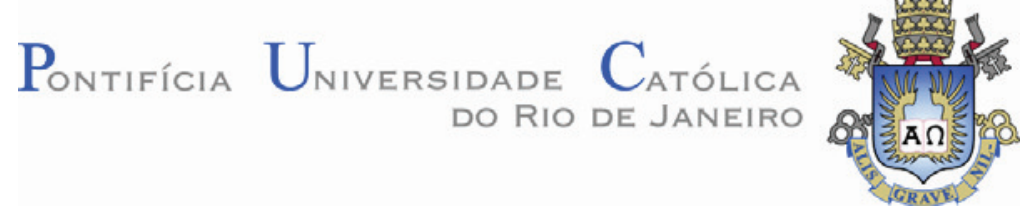

Eleazar Cristian Mejia Sanchez

Controle por Aprendizado Acelerado e Neuro-Fuzzy de Sistemas Servo - Hidráulicos de Alta Frequência

Dissertação de Mestrado

Dissertação apresentada como requisito parcial para obtenção do título de Mestre pelo Programa de PósGraduação em Engenharia Mecânica da PUC - Rio.

Orientador: Prof. Marco Antonio Meggiolaro

Rio de Janeiro, Setembro de 2009 


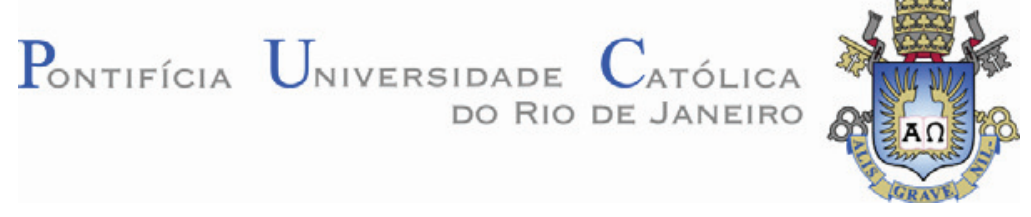

Eleazar Cristian Mejia Sanchez

\title{
Controle por Aprendizado Acelerado e Neuro-Fuzzy de Sistemas Servo - Hidráulicos de Alta Frequência
}

\begin{abstract}
Dissertação apresentada como requisito parcial para obtenção do título de Mestre pelo Programa de PósGraduação em Engenharia Mecânica da PUC - Rio. Aprovada pela Comissão Examinadora abaixo assinada.
\end{abstract}

Prof. Marco Antonio Meggiolaro

Orientador

Departamento de Engenharia Mecânica PUC - Rio

Prof. Jaime Tupiassu Pinho de Castro Departamento de Engenharia Mecânica PUC - Rio

Prof. ${ }^{a}$ Karla Tereza Figueiredo Leite Departamento de Engenharia Elétrica - PUC - Rio

Prof. José Eugenio Leal Coordenador Setorial do Centro Técnico Científico - PUC - Rio

Rio de Janeiro, Setembro 2009. 
Todos os direitos reservados. É proibida a reprodução total ou parcial do trabalho sem autorização da universidade, do autor e do orientador.

Eleazar Cristian Mejia Sanchez

Graduou-se em Engenharia Mecatrônica Universidad Nacional de Ingenieria Lima - Peru em 2005.

Ficha Catalográfica

Mejia Sanchez, Eleazar Cristian.

Controle por aprendizado acelerado e neuro-fuzzy de sistemas servo-hidráulicos de alta frequência / Eleazar Cristian Mejia Sanchez ; orientador: Marco Antonio Meggiolaro. - 2009.

130 f. : il.(color.) ; $30 \mathrm{~cm}$

Dissertação (Mestrado em Engenharia Mecânica)Pontifícia Universidade Católica do Rio de Janeiro, Rio de Janeiro, 2009.

Inclui bibliografia.

1. Engenharia mecânica - Teses. 2. Lógica fuzzy. 3. Sistemas de neuro-fuzzy. 4. Redes neurais. 5. Controle por aprendizado neuro-fuzzy. I. Meggiolaro, Marco Antonio. II. Pontifícia Universidade Católica do Rio de Janeiro. Departamento de Engenharia Mecânica. III. Título. 
Ao Senhor Jesus Cristo, meu pai Jesus Israel, minha mãe Agripina, meu irmão Ronald, e minha irmã Liliana, meus avós Marcos e Juana, e meus queridos amigos. 


\section{Agradecimentos}

Ao Professor Marco Antonio Meggiolaro pela paciência e orientação durante o desenvolvimento do curso de mestrado.

Agradecemos à Professora Karla Tereza Figueiredo Leite por toda a valiosa contribuição na concepção do controle proposto.

Ao Professor Jaime Tupiassú Pinho de Castro pelas sábias sugestões.

Ao meu grande amigo Juan Gerardo Castillo Alva, pela ajuda na parte experimental em todo momento.

A Marco Perez, Jesus Leal, Jorge Hinostroza e Gilmar amigos e colegas de laboratório.

Aos meus amigos Hernan zambrano, David Achanccaray, Nilton Anchayhua, Edwin Campos, Cesar Mamani, Josue e Carlos.

Aos professores da PUC - Rio pelo ensino.

Ao Departamento de Engenharia Mecânica da PUC - Rio e seus funcionários, pela colaboração comigo.

A CNPq Conselho Nacional de Desenvolvimento Cientifico e Tecnológico pela ajuda financeira.

A todas aquelas pessoas que de alguma outra forma participaram no desenvolvimento da dissertação. 


\section{Resumo}

Mejía Sánchez, Eleazar Cristian; Meggiolaro, Marco Antonio. Controle por Aprendizado Acelerado e Neuro-Fuzzy de Sistemas Servo-Hidráulicos de Alta Frequência. Rio de Janeiro, 2009. 130p. Dissertação de Mestrado Departamento de Engenharia Mecânica, Pontifícia Universidade Católica do Rio de Janeiro.

Nesta dissertação foram desenvolvidas técnicas de controle por aprendizado acelerado e Neuro-Fuzzy, aplicadas em um sistema servo-hidráulico para ensaio de fadiga. Este sistema tem o propósito de fazer ensaios em materiais para prever a resistência à fadiga dos materiais. O trabalho envolveu quatro etapas principais: levantamento bibliográfico, desenvolvimento de um controle por aprendizado acelerado, desenvolvimento de um controle por aprendizado Neuro-Fuzzy, e implementação experimental dos modelos de controle por aprendizado proposto em uma máquina de ensaios de materiais. A implementação do controle por aprendizado acelerado foi feita a partir do modelo de controle desenvolvido por Alva[5], com o objetivo de acelerar o processo de aprendizagem. Esta metodologia consiste em fazer um controle do tipo bang-bang, restringindo a servo-válvula a trabalhar sempre em seus limites extremos de operação, i.e., procurando mantê-la sempre completamente aberta em uma ou outra direção. Para manter a servo-válvula trabalhando em seus limites de seu funcionamento, os instantes ótimos para as reversões são obtidos pelo algoritmo de aprendizado, e armazenados em tabelas específicas para cada tipo de carregamento. Estes pontos de reversão dependem de diversos fatores, como a amplitude e carga média da solicitação, e são influenciados pela dinâmica do sistema. $\mathrm{Na}$ metodologia proposta, a lei de aprendizado inclui um termo de momentum que permite acelerar a aprendizagem dos valores das tabelas constantemente durante a execução dos testes, melhorando a resposta a cada evento. O desenvolvimento de um controle por aprendizado Neuro-Fuzzy foi motivado pela necessidade de ter um agente com a capacidade de aprendizado e armazenamento dos pontos ótimos de reversão. Este modelo de controle também consiste na implementação de um controle do tipo bang-bang, trabalhando com a servo-válvula sempre nos seus limites extremos de operação. O instante de reversão é determinado pelo sistema Neuro-Fuzzy, o qual tem como entradas a gama (dobro da amplitude) e o valor mínimo do carregamento solicitado. O processo de aprendizado é feito pelas 
atualizações dos pesos do sistema Neuro-Fuzzy, baseado nos erros obtidos durante a execução dos testes, melhorando a resposta do sistema a cada evento. A validação experimental dos modelos propostos é feita em uma máquina servohidráulica de ensaios de fadiga. Para este fim, o algoritmo de controle proposto foi implementado em tempo real em um módulo de controle CompactRIO da National Instruments. Os testes efetuados demonstraram a eficiência da metodologia proposta.

\section{Palavras-chave}

Sistemas Servo-hidráulicos; Controle de Alta Frequência; Controle por Aprendizado Acelerado; Controle Neuro-Fuzzy; Maquina de Testes de Fadiga. 


\section{Abstract}

Mejía Sánchez, Eleazar Cristian; Meggiolaro, Marco Antonio (Advisor). Accelerated Learning and Neuro-Fuzzy Control of High Frequency Servo-Hydraulic Systems. Rio de Janeiro, 2009. 130p. M.Sc. Dissertation - Departamento de Engenharia Mecânica, Pontifícia Universidade Católica de Rio de Janeiro.

In this thesis, accelerated learning and Neuro-Fuzzy control techniques were developed and applied to a servo-hydraulic system used in fatigue tests. This work involved four main stages: literature review, development of an accelerated learning control, development of a Neuro-Fuzzy control, and implementation of the learning control models into a fatigue testing machine. The accelerated learning control was implemented based on a learning control developed in previous works, introducing a faster learning law. Both learning control methodologies consist on implementing a bang-bang control, forcing the servovalve to always work in its operational limits. As the servo-valve works in its operational limits, the reversion points to achieve every peak or valley in the desired history are obtained by the learning algorithm, and stored in a specific table for each combination of minimum and mean load. The servo-valve reversion points depend on a few factors, such as alternate and mean loading components, while they are as well influenced by the system dynamics. In the proposed accelerated methodology, the learning law includes one momentum term that allows to speed up the learning process of the table cell values during the execution of the tests. The developed Neuro-Fuzzy control also consists on a bang-bang control, making the servo-valve work in its operational limits. However, here the instant of each reversion is determined by the Neuro-Fuzzy system, which has the load range and minimum load required as inputs. The learning process is made by the update of the Neuro-Fuzzy system weights, based on the errors obtained during the execution of the test.The experimental validation of the proposed models was made using a servo-hydraulic testing machine. The control algorithm was implemented in real time in a C-RIO computational system. The tests demonstrated the efficiency of the proposed methodology.

\section{Keywords}

Servo-Hydraulic Systems; High Frequency Control; Accelerated Learning Control; Neuro-Fuzzy Control; Fatigue Testing Machine. 


\section{Sumário}

$\begin{array}{ll}1 . \text { Introdução } & 17\end{array}$

$\begin{array}{ll}\text { 1.1. Objetivo } & 17\end{array}$

1.2. Considerações Iniciais $\quad 17$

1.3. Motivação 18

1.4. Revisão Bibliográfica 20

1.5. Roteiro da Dissertação 22

2 Lógica Fuzzy 23

2.1. Introdução 23

2.2. Conjuntos Fuzzy 24

2.2.1. Variáveis lingüísticas $\quad 26$

2.2.2. Função de Pertinência. 27

2.2.3. Operações entre Conjuntos Fuzzy 28

2.3. Sistema de Inferência Fuzzy 30

2.3.1. Fuzzificação 30

2.3.2. Regras e Inferência Fuzzy 31

2.3.3. Defuzzificação.

2.4. Tipos de Sistema Fuzzy 32

2.4.1. Modelo de Mandani 33

2.4.2. Modelo de Takagi-Sugeno 33

2.5. Vantagens e Desvantagens dos Sistemas Fuzzy 34

2.5.1. Vantagens dos Sistemas Fuzzy 34

2.5.2. Desvantagens dos Sistemas Fuzzy 35

3 Redes Neurais 36

3.1. Introdução 36

3.2. Estrutura do neurônio $\quad 37$

3.2.1. Peso de conexão 38

3.2.2. Função Somatório (Net) 38

3.2.3. Função de Ativação 39 
3.3. Arquitetura das Redes Neurais 41

3.3.1. Rede Perceptron 41

3.3.2. Rede Multilayer Perceptron 43

3.4. Algoritmo Backpropagation 48

3.4.1. Fases do Algoritmo Backpropagation 48

3.4.2. Algoritmo de Aprendizado 50

3.4.3. Parâmetros de Aprendizado 54

3.5. Modelagem da ANN 57

3.6. Vantagens e Desvantagens das ANN 58

3.6.1. Vantagens das $A N N \quad 58$

3.6.2. Desvantagens das ANN 58

4. Sistemas Neuro-Fuzzy 59

4.1. Introdução 59

4.2. Sistemas Híbridos 60

4.2.1. Sistema Híbrido Seqüencial 60

4.2.2. Sistema Híbrido Auxiliar 60

4.2.3. Sistema Híbrido Incorporado 61

4.3. Sistemas Neuro-Fuzzy 62

4.3.1. Características de Sistemas Neuro-Fuzzy 63

4.3.2. Modelos de Sistemas Neuro-Fuzzy 66

4.4. Vantagens e Desvantagens dos SNF 72

4.4.1. Vantagens das SNF 72

$\begin{array}{ll}\text { 4.4.2. Desvantagens dos SNF } & 72\end{array}$

5. Controle por Aprendizado Acelerado e Simulação 73

5.1. Introdução 73

5.2. Modelagem do Sistema Servo-Hidráulico 73

5.2.1. Modelagem da Servo-válvula 74

5.2.2. Modelagem do Pistão Hidráulico 76

5.2.3. Modelos lineares $\quad 77$

5.3. Controle por Aprendizado Acelerado 79

5.3.1. Metodologia de controle 80

5.3.2. Tabelas de Aprendizado $\quad 81$ 
5.3.3. Determinação do Valor de $\bigcup_{I J}$

5.3.4. Processo de Aprendizado 85

5.3.5. Algoritmo de Aprendizado 90

5.3.6. Resultado das simulações do Algoritmo de Controle por Aprendizado Acelerado

6. Controle por Aprendizado Neuro-Fuzzy 97

6.1. Introdução 97

6.2. Esquema do controle por aprendizado NF 97

6.3. Modelagem do Controle por Aprendizado Neuro-Fuzzy 99

6.3.1. Modelagem Fuzzy do SNF 99

6.3.2. Modelagem da parte neural 101

6.4. Controle por Aprendizado Neuro-Fuzzy 102

6.4.1. Calculo do valor de $U_{I J}$

6.4.2. Lei de aprendizado do SNF 104

6.4.3. Algoritmo de controle por aprendizado Neuro-Fuzzy 105

6.4.4. Resultado das simulações do controle por aprendizado SNF 107

7. Resultados Experimentais 119

7.1. Sistema Experimental 119

7.2. Modulo de Controle CompactR/O 121

7.3. Software desenvolvido em LabVIEW 123

7.4. Resultados Experimentais 124

8. Conclusões 128

$\begin{array}{ll}\text { Bibliografia } & 129\end{array}$ 


\section{Lista de figuras}

Figura 1.1. Ensaio de Fadiga. 19

$\begin{array}{lll}\text { Figura 2.1. (a) lógica booleana } & \text { (b) lógica fuzzy. } & 25\end{array}$

Figura 2.2. Variável lingüística 26

Figura 2.3. Funções de pertinência. $\quad 27$

Figura 2.4. Funções de pertinência: (a) Triangular (b) Trapezoidal

(c) Sigmoidal (d) Função de Bell. 28

Figura 2.4. Operações entre conjuntos fuzzy: (a) Conjuntos A e B,

(b) União, (c) Interseção e (d) Complemento. [2]. 29

Figura 2.5. Sistema de Inferência Fuzzy. 30

Figura 2.6. Regra e inferência fuzzy [8]. 31

Figura 2.7. Processo de Defuzzificação. 32

Figura 3.1. Esquema básico do neurônio biológico. 37

Figura 3.2. Modelo básico de um neurônio Artificial "Perceptron". 38

Figura 3.3. Funções de Ativação: (a) Função de Grau. (b) Função linear.

(c) Função Logsig. (d) Função Tansig.

Figura 3.4. Rede perceptron com $k$ Neurônios de saída. 41

Figura 3.6. Configuração da rede MLP. 45

Figura 3.7. Treinamento Supervisionado. 48

Figura 3.9. (a) Fase de propagação (b) Sinais Funcionais. 49

Figura 3.10. (a) Fase de Retro-propagação (b) Sinais de erro. 50

Figura 3.11. Cálculo de erro $e_{j}$ na camada de saída. 53

Figura 3.13. Taxa de aprendizado pequeno, com problema do mínimo local. 55

Figura 3.14. Taxa de aprendizado grande, com problema de oscilações. $\quad 55$

Figura 3.15. Aprendizado com Termo de Momentum. 56

Figura 4.1. Sistema Híbrido Seqüencial. 60

Figura 4.2. Sistema Híbrido Auxiliar. 61

Figura 4.3. Sistema Hibrido Incorporado. 61

Figura 4.4. Arquitetura básica de um Sistema Neuro-Fuzzy. 62

Figura 4.5. Características do Sistema Neuro-Fuzzy. 63

Figura 4.6. Características fuzzy do Sistema Neuro-Fuzzy. 64 
Figura 4.7. Características RNA do Sistema Neuro-Fuzzy.

Figura 4.8. Arquitetura ANFIS. 66

Figura 4.9. Arquitetura NEFCLASS. 69

$\begin{array}{ll}\text { Figura 4.10. Arquitetura FSOM. } & 70\end{array}$

Figura 5.1. Sistema Servo-Hidráulico

Figura 5.2. Representação esquemática da servo-válvula. $\quad 74$

Figura 5.3. Diagrama de blocos do controle por aprendizado. 80

Figura 5.4. Pontos de reversão da servo-válvula. $\quad 81$

Figura 5.5. Termo de momentum em função do erro para $a=10$ e $b=0,5 . \quad 87$

Figura 5.6. Esquema do grau de influência do erro. $\quad 88$

Figura 5.7. Algoritmo de controle por aprendizado acelerado. 91

Figura 5.8. Resposta do controle por aprendizado acelerado para um

carregamento de amplitude constante de $\pm 10 \mathrm{kN}$. 92

Figura 5.9. Resposta do controle por aprendizado acelerado para um carregamento de amplitude constante de $\pm 75 \mathrm{kN}$.

Figura 5.10. Resposta do controle por aprendizado acelerado para

diferentes amplitudes de carregamento.

Figura 5.11. Resposta do controle por aprendizado acelerado para carregamento já apresentados.

Figura 5.12. Resposta para um carregamento constante de $\pm 25 \mathrm{kN}$ : (a) Controle por aprendizado de Alva e

(b) Controle por aprendizado acelerado.

Figura 5.13. Erro do controle por aprendizado de Alva e

aprendizado acelerado. 96

Figura 6.1. Diagrama de blocos do controle por aprendizado Neuro-Fuzzy. $\quad 98$

Figura 6.2. Estrutura do Sistema Neuro-Fuzzy. 98

Figura 6.3. Função de pertinência triangular do $S N F$. 100

Figura 6.4. Particionamento Fuzzy Grid, onde $\rho_{1}, \gamma_{1}, \mu_{1}$ e $\rho_{2}, \gamma_{2}, \mu_{2}$, são os graus de pertinência dos conjuntos Fuzzy das variáveis " minimo" e "gama" respectivamente.

Figura 6.5. Calculo de $U_{\text {IJ }}$ e descrição das camadas do SNF. 103

Figura 6.6. Algoritmo de controle por aprendizado Neuro-Fuzzy. 106

Figura 6.7. Resposta do controle por aprendizado Neuro-Fuzzy 
para um carregamento de amplitude constante de $\pm 10 \mathrm{kN}$.

Figura 6.8. Resposta do controle por aprendizado Neuro-Fuzzy para

um carregamento de amplitude constante de $\pm 75 \mathrm{kN}$.

Figura 6.9. Resposta do controle por aprendizado Neuro-Fuzzy para

diferentes amplitudes de carregamento.

Figura 6.10. Resposta do controle por aprendizado Neuro-Fuzzy para carregamentos já apresentados anteriormente.

Figura 6.11. Desempenho dos Modelos de controle por aprendizado

para carregamento constante $\pm 20 \mathrm{kN}$.

Figura 6.12. Desempenho do controle por aprendizado para $\eta=0.1 \mathrm{e}$ carregamento de amplitude constante de $\pm 25 \mathrm{kN}$.

Figura 6.13. Desempenho do controle por aprendizado para $\eta=0.95 \mathrm{e}$ carregamento de amplitude constante de $\pm 25 \mathrm{kN}$.

Figura 6.14. Desempenho do controle por aprendizado para $\eta=1.5 \mathrm{e}$ carregamento constante de $\pm 25 \mathrm{kN}$.

Figura 6.15. Número de ciclos de convergência em função da taxa de aprendizado, carregamento de $\pm 25 \mathrm{kN}$.

Figura 6.16. Desempenho do controle por aprendizado para $\eta=0.1 \mathrm{e}$ carregamento de amplitude constante de $\pm 80 \mathrm{kN}$.

Figura 6.17. Desempenho do controle por aprendizado para $\eta=0.85$ e carregamento de amplitude constante de $\pm 80 \mathrm{kN}$.

Figura 6.18. Desempenho do controle por aprendizado para $\eta=0.95 \mathrm{e}$ carregamento de amplitude constante de $\pm 80 \mathrm{kN}$.

Figura 6.19. Número de ciclos de convergência em função da taxa de aprendizado, carregamento de $\pm 80 \mathrm{kN}$.

Figura 6.20. Número de ciclos de convergência em função da taxa de aprendizado, para diferentes carregamentos.

Figura 7.1. Máquina de Ensaios INSTRON 8501.

Figura 7.2. Conexões para o sistema de controle por aprendizado.

Figura 7.3. Controlador $c R I O-9004$.

Figura 7.4. Componentes do Chip FPGA.

Figura 7.5. (a) NI cRIO 9263 e (b) NI cRIO 9237. 
Figura 7.6. Tela de controle por aprendizado Neuro-Fuzzy.

Figura 7.7. Resposta da máquina servo-hidráulica a um ensaio de fadiga sob amplitude constante com controle por aprendizado Neuro-Fuzzy. 124

Figura 7.8 Convergência do erro em função do numero de ciclos.

Figura 7.9. Antecipação dos pontos de reversão à medida que a

frequência é aumentada. 


\section{Lista de tabelas}

Tabela 1. Tabela de aprendizado. $\quad 82$

Tabela 2. Tabelas de aprendizado e de momentum. 83

Tabela 3. Método de interpolação quando os valores de gama e

mínimo estão entre duas células. 